\title{
Combining Wavelet Saliency, Color and DCT Coefficients for Content-Based Image Retrieval
}

\author{
Alberto Rios Júnior ${ }^{1}$ and Díbio Leandro Borges ${ }^{2}$ \\ 1 Department of Mechanical Engineering \\ University of Brasília, Brasília, DF 70910-900, Brazil \\ albertoriosjr@gmail.com \\ 2 Department of Computer Science \\ University of Brasília, Brasília, DF 70910-900, Brazil \\ dibio@unb.br
}

\begin{abstract}
This paper presents an approach for content-based image retrieval extracting salient points and regions from images, and also aggregating color and DCT values in a signature descriptor for recognition. Salient points and regions are extracted from each image by a wavelet decomposition over the color channels where the highest coefficients in coarsest levels are the centers of salient regions in finest resolution. These local regions are support for extracting color histograms and a set of DCT magnitudes in order to derive a signature for the image. A feature vector combining histograms of color channels and DCT values is proposed and tested as signature of the image. Public COIL, Caltech, and $\mathrm{ZuBuD}$ images datasets are used for testing. Results comparing variations of the descriptor based on wavelet saliency are given on all those image datasets supporting potential for the proposed method.
\end{abstract}

Keywords: content-based image retrieval, wavelet salient points, global signature, category recognition.

\section{Introduction and Related Work}

Content-Based Image Retrieval considers the problem of querying a database with visual data and retrieving from it the most similar visually images. An image may be similar to others in a multitude of aspects, and finding a particular set of features that helps measuring its similarity is a challenging task in Computer Vision. In this work we are interested in features that are compact and appear in places with more relative information than others. Salient points are these features 4, however from the literature it is a major research problem providing an effective saliency detection and related descriptors for general visual image retrieval tasks. We propose a saliency detection which takes into consideration color in a wavelet based decomposition and selection process, and also a signature descriptor together with distance formulations for image retrieval.

There are two major strategies for using salient points for recognition: Local and Global salient signatures. A survey of important works as local signature

E. Bayro-Corrochano and E. Hancock (Eds.): CIARP 2014, LNCS 8827, pp. 917-924 2014.

(C) Springer International Publishing Switzerland 2014 
approaches can be seen in 9 . Generally in this strategy after the salient points are localized a set of feature signatures is built in order to provide ways of feature to feature matching. SIFT [7, is a mostly cited work in the local strategy.

In the global strategy after the salient points are detected a signature is built using all neighborhoods, and all images are compared based on that vector. One of the earliest works in this approach is [6], where it is proposed to use a wavelet decomposition of a luminance image, at each scale the largest magnitude coefficients are kept recursively till the last scale desired. The feature vectors proposed were HSV mean, variance and skewness over 3x3 windows in [13], and color moments and gabor filters in [1]. In [4] a wavelet based saliency detection is also proposed selecting the largest magnitude coefficients from the luminance channel. The signature is built using a color constancy model [3] and a texture bank with rotated wavelet filters.

It is proposed in this paper a salient point detection and global signature for image retrieval which is novel in a wavelet based saliency algorithm that includes and evaluates the color channels for the saliency computation and selection, and also in building a new feature vector as a combination of HS histograms and DCT values in neighborhoods around the salient points.

\section{Salient Points Detection by Wavelet Analysis}

An image brings extensive information on the objects and their interactions with light. The optical sensing system used to capture the image and its viewpoint have also big influences on the visual information pictured in the image. On top of that the singularities of an image signal change with scale, so in order to find more distinguished and repetitive points useful for signature and recognition it is important to consider scales [5].

The wavelet representation gives important details on distinct signal scales [8], and therefore the wavelet discrete transformation can be useful to extract salient points throughout different scales of an image. High wavelet coefficients lead to regions with high global variations on signal. Based on this it was firstly proposed in [6] a method to find salient points tracking the highest wavelet coefficients in all scales. The method uses the discrete wavelet transform as a tool for multi-scale analysis. The input image is analyzed on scales $2^{-j}, j \in \mathbb{Z}^{+}$. Due to the compact support of the used wavelet transformation it is possible to track from what points a coefficient was derived in a scale $2^{j}$. This set of points in scale $2^{j+1}$ derived from coefficients in $2^{j}$ is defined as children of the coefficients in $W_{2 j} f(n)$ (equation 1).

$$
C\left(W_{2 j} f(n)=W_{2^{j+1}} f(k), 2 n \leq k \leq 2 n+p-1\right.
$$

From the children of each coefficient, a support region can be determined. Among the points in this region, one with the highest absolute values is selected for a more detailed analysis. At the final stage the image gradient is also computed. High wavelet coefficients with high gradient values will indicate most salient 


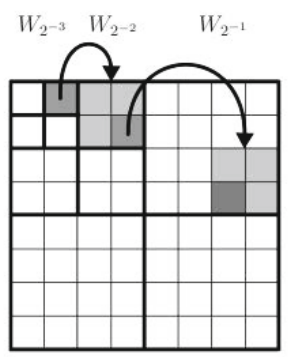

Fig. 1. Coefficients and their relation through scales in the saliency detection

points (Fig. 1). The saliency map is built from the sum of the absolute values in the tracked path (equation 2).

$$
\text { saliency }=\sum_{k=1}^{-j}\left|C^{(k)}\left(W_{2 j} f(n)\right)\right|,-\log _{2} N \leq j \leq-1
$$

\subsection{Wavelet Based Saliency Considering Colors}

One of the limitations of the earlier method in [6] is that it only considers one channel (the luminance) of the image. Later, in [13] an extension for using color after the salient points was presented. However, the color information was not considered in the selection of the salient points.

Here we propose to compute a wavelet based saliency map considering color channels from the start. In each layer (e.g. RGB) of the initial image a wavelet discrete transformation is applied and a set of the highest salient points for each is selected. The mean of the highest selected of each layer is computed and in the end only the highest means for the 3 channels are considered for the final saliency map. Interesting different points are included in this new process. This process is detailed by the Algorithm 1, wherein calculateSaliency () is mainly the implementation of the earlier [6] method.

In order to verify the performance of this new extraction scheme we designed experiments considering three variations of the Wavelet Saliency Descriptor: 1) WSDLum (Wavelet Saliency Descriptor on Luminance), when the input image is first converted to gray before saliency calculation; 2) WSDColor RGB (Wavelet Saliency Descriptor on RGB color channels), when the input image is used in RGB color model; 3) WSDColorInv (Wavelet Saliency Descriptor on Invariant Color Space), when the image is converted to the $\mathrm{c} 1 \mathrm{c} 2 \mathrm{c} 3$ color space, for color invariance testing [3]. Fig. 2] shows examples of the extracted salient points and regions, wherein these regions were extracted considering the three variations of detection, WSDLum, WSDColor RGB, WSDColorInv. It is shown only the $10 \%$ most salient, but this is a control parameter of the algorithm. It is possible to notice that results are spread around interesting parts of the image and objects, 


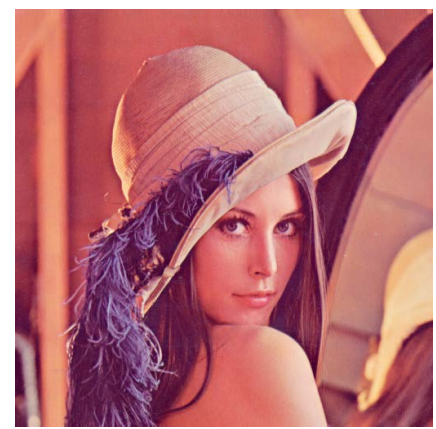

(a)

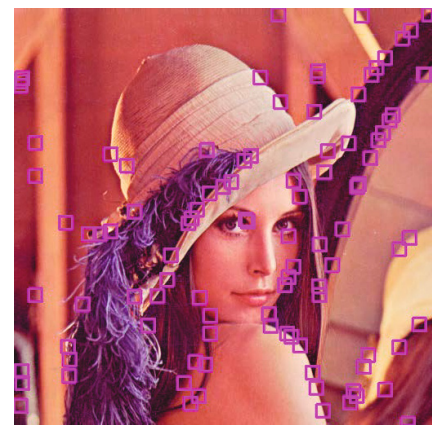

(c)

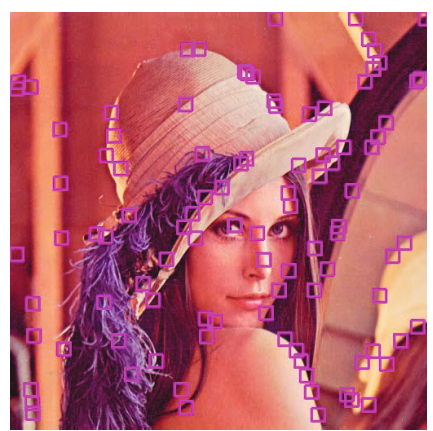

(b)

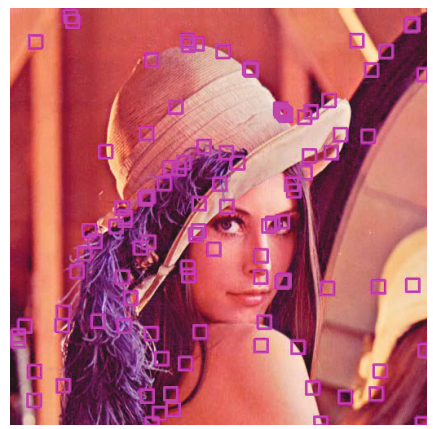

(d)

Fig. 2. Typical image (Lenna) with selected salient points and regions ( $10 \%$ highest): (a) original, (b) WSDLum, (c) WSDColorRGB, (d) WSDColorInv

and the use of color channels, either RGB direct or c1c2c3 invariant, makes a difference in the selected regions.

\subsection{Combining Salient Points, Color and DCT Coefficients}

As derived from the Wavelet Saliency Detection proposed we gathered a set of distinguished marks (salient points) in the image, ranked according to their distinctiveness. These will be loci of visual attention and the elements surrounding those points could be used for deriving descriptors for the image. We propose here a signature descriptor that will combine elements from those regions of attention. A special combination of color frequencies in HSV domain and the highest DCT coefficients from regions surrounding the salient points composes the descriptor. The feature vector is (H_bins, S_bins, $N_{-} D C T \_$values).

The Color Histogram Part of the Descriptor. This work proposes the use of a histogram built from the color hue and saturation channels. Using 32 bins for $\mathrm{H}$, and 30 for $\mathrm{S}$ captured the color variation and histogram intersection was a metric for comparing this part of the descriptor. 


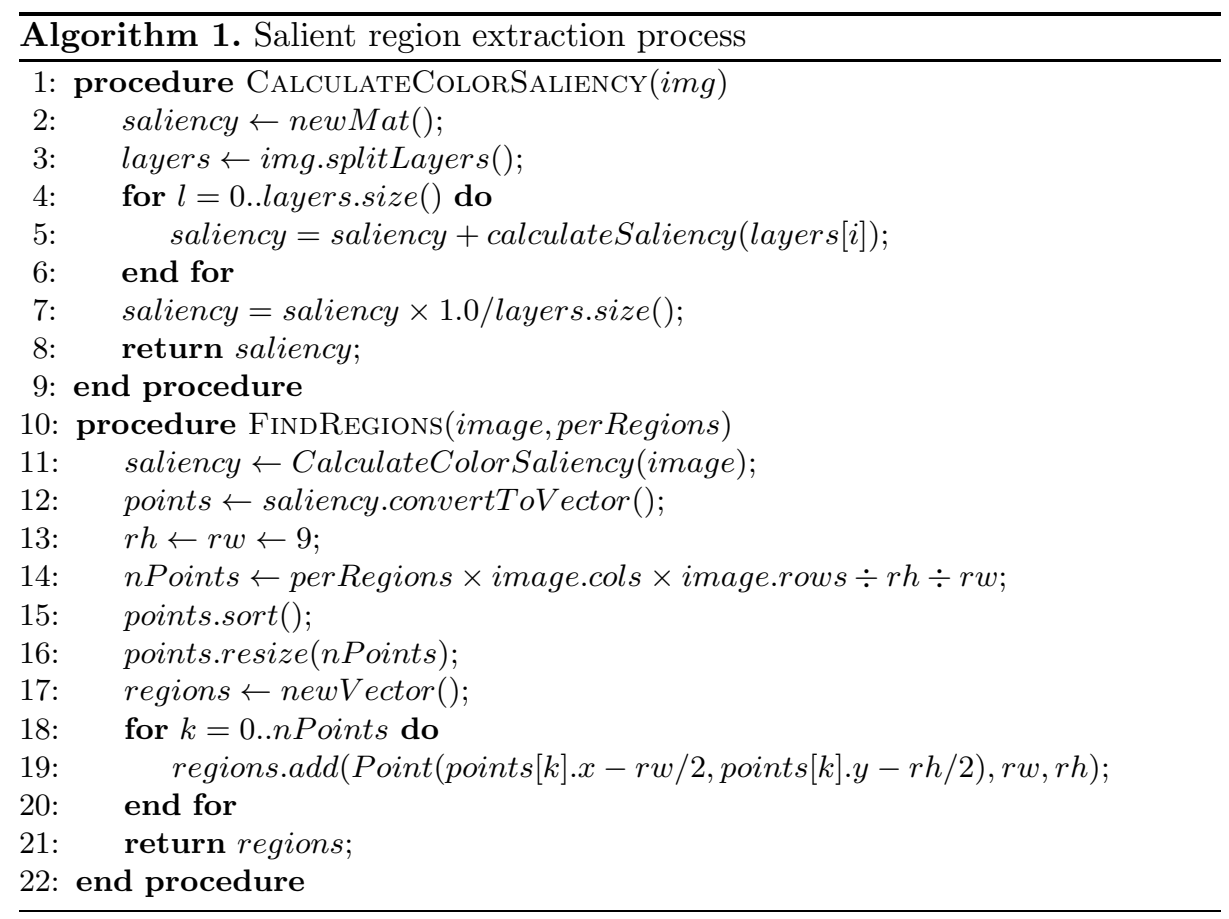

The DCT Coefficients Part of the Descriptor. The DCT (Discrete Cosine Transform) values can capture visual texture information [1]. We propose DCT coefficients as part of the descriptor, normalized to the interval $[0,1]$. Later, the highest values are selected as features of the salient regions. The difference between two DCT vectors can be calculated by equation 3 .

$$
D_{D}(i, j)=\left\|1-\frac{i^{T} j}{\left\|d_{j}\right\|\|q\|}\right\|
$$

Combining Distance Functions. For evaluating the similarity of the feature vector we propose to combine the distance on color histograms and the DCT distance. A way to combine those distances is using the mean between the two distance metrics. Thus the distance between two images is given by equation 4 wherein $D_{N}(K, M)$ is the difference between two color histograms and $D_{D}(K, M)$ is the difference between DCT coefficients.

$$
D(K, M)=\frac{D_{N}(K, M)+D_{D}(K, M)}{2}
$$

Varying the Number of Salient Points and Regions. It is possible to choose a percentage of an image area to select the number of salient points from. The window size around a salient point can be chosen, so the number 
of salient points is given by percentage $\times$ image.cols $\times$ image.rows $\div r h \div r w$, wherein rh is region's height and rw is region's width. We tested variations of the percentage and window sizes to show performance and to choose the best parameters for the databases.

\section{Results and Discussion}

Experiments on the proposed Wavelet Saliency Descriptor were run on three public image datasets which pose different challenges for image retrieval. Tests measuring recall $\mathrm{x}$ precision rates were gathered for the following conditions: $1 \%, 5 \%$, and $10 \%$ most salient points for WSDLum, WSDColorRGB, and $W S D C o l o r I n v$; and also varying for the same conditions the size of the regions surrounding those points, 9x9, 16x16, and 32x32. Values for $H_{\text {_bins }}=30$, S_bins $=32$, and N_DCT_values $=100$, window $16 \times 16$ and $10 \%$ highest saliency provided the best results. To summarize the results the best figures are shown and comments on the conditions are given.

Public Image Databases. In order to evaluate the proposal three public known image databases were used. COIL-100 consists of 100 objects with flat uniform background. There are 72 images per object. It is largely used to check performance of visual retrieval techniques, especially because there is a reasonable variation of viewpoints of the objects [10. Zurich Buildings Image Database $(\mathrm{ZuBuD})[12$ is another common image database used to evaluate retrieval algorithms. The base contains pictures of 201 buildings in Zurich (5 images per scene). Images were taken under natural illumination and provide a different set of objects to be tested. The Caltech Categories database [2] is a benchmark dataset for image retrieval methods. It contains 3.649 images in 7 categories. Images of objects are shown at very diverse and common situations, especially regarding objects with different backgrounds. For testing, we split the images in two sets, queries and results, and depending on the class and the database the number of images in each set varied.

Retrieval Results. Figure 3 shows recall x precision curves for all datasets comparing the variations of salient detection and descriptors. The salient points, in WSDLum, are detected from a gray level (i.e. luminance) channel only. $W S D C$ olor $R G B$ considers all 3 channels (RGB) in separate, extract salient points from each and then average the results and select the highest means. $W S D$ ColorInv works similarly to $W S D C$ olor $R G B$ but on c1c2c3 channels instead of RGB. Overall the retrieval results on the three datasets showed high performance, reaching precision rates above 0.7 for recall bigger than 0.6 in the case of WSDLum and WSDColor RGB in COIL-100 (Fig. 3(a)), and precision above 0.7 with recall 0.5 for WSDLum, WSDColor RGB, and WSDColor Inv for both COIL-100 and ZuBuD datasets (Fig. 3(a) and 3(b)). All precision rates were above 0.5 for recall 0.3 , in Caltech Categories. It is ought to remember that the proposed descriptors do not perform geometric matching between images, 
only a vector signature distance calculation, and the reached recognition figures on these datasets are highly promising. The use of the color channels for the saliency detection improved the performance of recognition on all three datasets, in COIL-100 and Caltech Categories WSDColor RGB was better consistently (Fig. 3(a), 3(c), and 3(d)), and in $\mathrm{ZuBuD}$ where all images were taken outdoor $W S D C o l o r I n v$ performed better (Fig. 3(b)) than the other two.

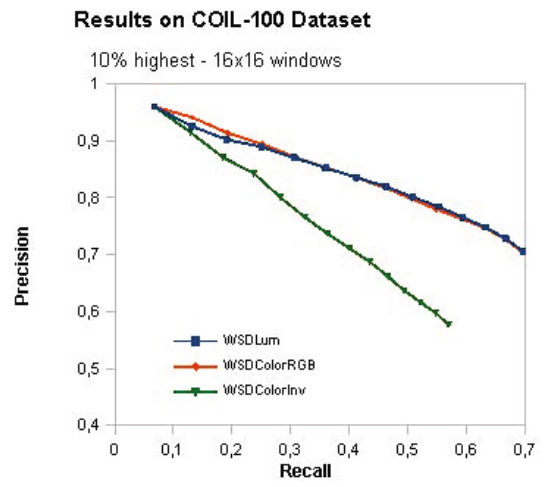

(a)

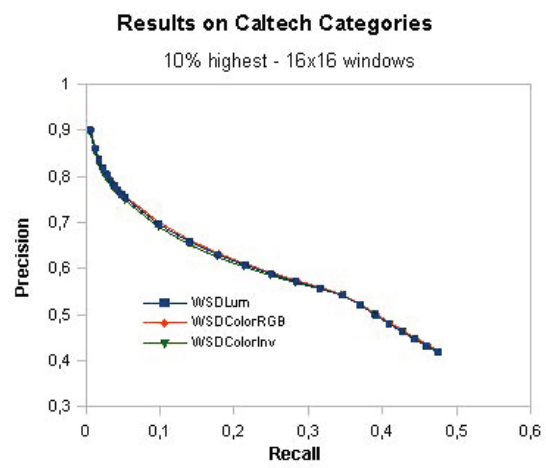

(c)

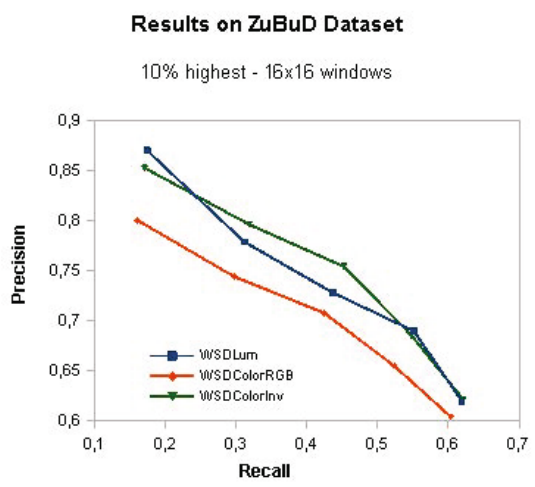

(b)

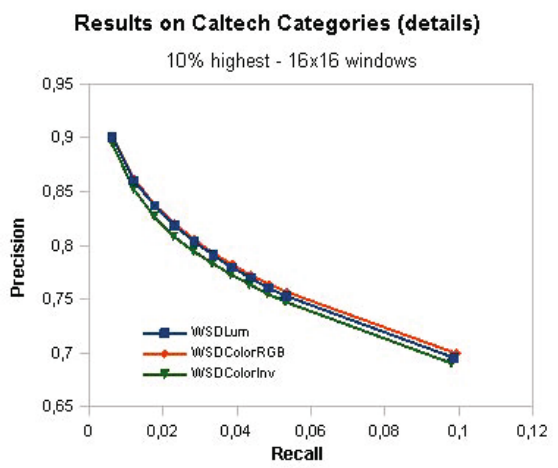

(d)

Fig. 3. Results of WSDLum, WSDColor RGB, and WSDColorInv on the Caltech Categories. (a) Caltech Categories; (b) Detail on Caltech Categories.

\section{Conclusions and Future Work}

A method to extract salient points from images, based on wavelet analysis and considering the three color channels from the image signal was presented. The method extends earlier work in [6] for color channels. Also, a local descriptor combining HS histograms and DCT coefficients in surrounding regions of the 
selected points was proposed and tested in visual retrieval tasks. A metric for deciding upon the similarity of feature vectors of images fusing color histograms and DCT coefficients was proposed and tested.

Results were given for typical visual image retrieval in three public databases with high and promising recognition rates. Although less than $10 \%$ higher in precision, the color descriptors were better consistently, and the feature vector for all three versions were using color information in all cases. Future work will consider local and geometric matching for object to object recognition, and also testing the inclusion of other features to the descriptor.

Acknowledgments. This research was partially supported by FINATEC and $\mathrm{DPP} / \mathrm{UnB}$. We thank our colleagues from the PPMEC/UnB for academic support.

\section{References}

1. Bimbo, A.D.: Visual Information Retrieval. Morgan Kaufmann (1999)

2. Caltech: Caltech Categories Site (2014),

http://www.vision.caltech.edu/html-files/archive.html (accessed May 4, 2014)

3. Geusebroek, J., Van den Boomgaard, R., Smeulders, A., Geerts, H.: Color invariance. IEEE Trans. on PAMI 23(12), 1338-1350 (2001)

4. Laurent, C., Laurent, N., Maurizot, M., Dorval, T.: In depth analysis and evaluation of saliency-based color image indexing methods using wavelet salient features. Multimedia Tools and Applications 31(1), 73-94 (2006)

5. Liu, Y., Zhang, D., Lu, G., Ma, W.Y.: A survey of content-based image retrieval with high-level semantics. Pattern Recognition 40(1), 262-282 (2007)

6. Loupias, E., Sebe, N., Brest, S., Jolion, J.: Wavelet-based salient points for image retrieval. In: ICIP 2000, vol. 2, pp. 518-521. IEEE (2000)

7. Lowe, D.: Distinctive image features from scale-invariant keypoints. Int. J. of Computer Vision 60(2), 91-110 (2004)

8. Mallat, S.: A theory for multiresolution signal decomposition: the wavelet representation. IEEE Trans. on PAMI 11(7), 674-693 (1989)

9. Mikolajczyk, K., Schmid, C.: A performance evaluation of local descriptors. IEEE Trans. on PAMI 27(10), 1615-1630 (2005)

10. Nayar, S., Nene, S., Murase, H.: Columbia object image library (coil 100). Tech. rep., Dept Computer Science, Columbia University, USA (1996)

11. Sebe, N., Lew, M.: Comparing salient point detectors. Pattern Recognition Letters 24(1), 89-96 (2003)

12. Shao, H., Svoboda, T., Van Gool, L.: Zubud: zurich buildings database for image based recognition. Tech. rep., Computer Vision Lab, Swiss Federal Institute of Technology, Switzerland (2003)

13. Tian, Q., Sebe, N., Loupias, E., Huang, T., Lew, M.: Image retrieval using wavelet-based salient points. Journal of Electronic Imaging 10(4), 835-849 (2001) 